03.1

\title{
Влияние на аномальную интенсификацию отрывного турбулентного течения угла наклона однорядных овально-траншейных лунок на стабилизированном гидродинамическом участке узкого канала
}

\author{
(C) С.А. Исаев ${ }^{1-3}$, А.Б Мазо ${ }^{3}$, Д.В. Никущенко ${ }^{1}$, И.А. Попов ${ }^{4}$, А.Г. Судаков ${ }^{2}$ \\ ${ }^{1}$ Санкт-Петербургский государственный морской технический университет, Санкт-Петербург, Россия \\ ${ }^{2}$ Санкт-Петербургский государственный университет гражданской авиации, Санкт-Петербург, Россия \\ ${ }^{3}$ Казанский (Приволжский) федеральный университет, Казань, Россия \\ ${ }^{4}$ Казанский национальный исследовательский технический университет им. А.Н. Туполева - Казанский \\ авиационный институт, Казань, Россия \\ E-mail: isaev3612@yandex.ru
}

Поступило в Редакцию 6 июля 2020 г.

В окончательной редакции 22 июля 2020 г.

Принято к публикации 24 июля 2020 г.

Наклон овально-траншейной лунки в коридорном пакете на стабилизированном гидродинамическом участке узкого канала оказывает кардинальное воздействие на аномальную интенсификацию отрывного и закрученного потоков в лунке, приводя к трех-четырехкратному снижению относительного отрицательного трения в диапазоне изменения углов от 40 до $60^{\circ}$, увеличению наибольшей скорости возвратного и вторичного течения до 0.8 и 1.18 по отношению к среднемассовой скорости потока в канале.

Ключевые слова: наклоненные овально-траншейные лунки, узкий канал, интенсификация.

DOI: 10.21883/PJTF.2020.21.50190.18454

Вихревые генераторы для интенсификации процессов переноса в пристеночных турбулентных течениях представляют большой интерес для разработки перспективных технологий в энергетике [1,2]. Среди поверхностных вихрегенераторов выделяются лунки, позволяющие добиваться значительной тепловой эффективности при весьма умеренных гидравлических потерях. В большинстве исследований рассматривались симметричные, в частности сферические, формы лунок как наиболее технологичные при их нанесении на стенку. Однако стремление интенсифицировать отрывное течение внутри лунок и спиралевидные вихри в следе за лунками обусловливает давний интерес к несимметричным формам лунок, в частности формирующим асимметричное течение. Как показано в [3], при выдуве струи под косым углом к сносящему потоку отмечается увеличение скорости течения в развивающихся спиралевидных вихревых структурах. Аналогичное воздействие на пристеночное течение оказывает изменение угла ориентации вихревых генераторов флюгерного типа [4]. В [5] интенсификация вторичного течения в лунке была достигнута при замене боковой половины сферической лунки на эллиптическую. Более эффективной оказалась наклонная к внешнему потоку овальная лунка, в которой две разнесенные половинки сферической лунки соединяются цилиндрической, траншейной вставкой [6]. В [7] исследованы метастабильные вихревые структуры в глубоких сферических и овальных расположенных поперек потока лунках. Показано, что забор среды в глубокую овальную траншею может происходить либо поочередно в области концов лунки с выходом наружу у противоположных концов (сферические лунки и короткие траншеи), либо одновременно с обоих концов (умеренные и длинные траншеи) с выходом в спутный поток из срединной части траншеи. При наклоне под углом $45^{\circ}$ увеличение длины уединенной овально-траншейной лунки (ОТЛ) на стенке узкого канала при условии постоянства площади пятна и глубины приводит к интенсификации вихревого течения в лунке и снижению гидравлических потерь $[8]$. В $[9,10]$ открыто явление аномальной интенсификации отрывного течения в наклонных однорядных ОТЛ в узком канале на стабилизированном гидродинамическом участке. Установлен многократный рост абсолютной величины относительного трения в отрывной зоне на участке забора среды в лунке. Максимальная скорость возвратного течения в лунках приближается к среднемассовой скорости, а максимальная скорость вторичного течения превышает максимальную скорость в плоскопараллельном канале. Эффект усиливается по мере уплотнения луночного пакета. В настоящей работе анализируется влияние на эффект угла $\theta$ наклона ОТЛ в однорядном пакете на стабилизированном гидродинамическом участке канального потока в диапазоне изменения $\theta$ от 0 до $65^{\circ}$.

Рассматривается периодическая секция канала с одной лункой на стенке длиной 8 , шириной 9 и высотой 1 (рис. 1). Верхняя стенка канала и боковые грани плоские. Лунка имеет ширину 1.05 , длину 7.05 , глубину 0.3 и радиус скругления кромки 0.21. Угол наклона лунки варьируется от 0 до $65^{\circ}$. Число Рейнольдса, определенное по характерной среднемассовой скорости и высоте канала, задано равным $10^{4}$. Декартова система коорди- 


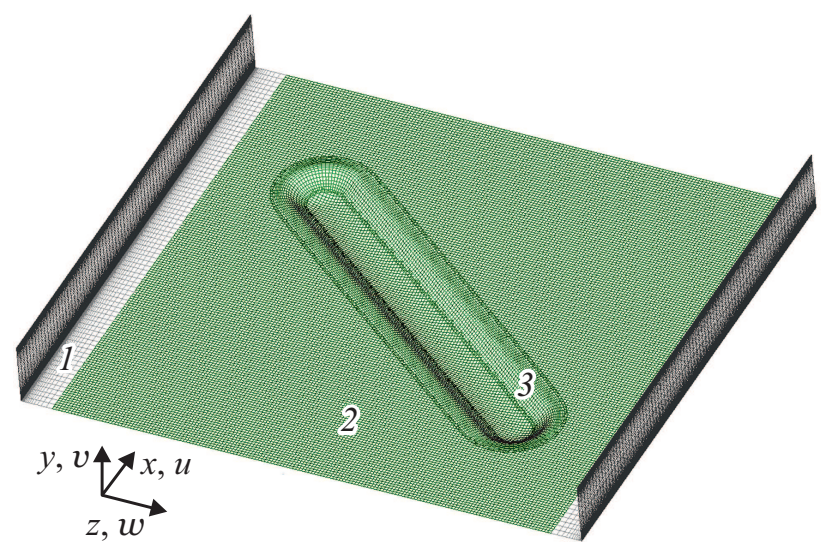

Рис. 1. Конфигурация периодической секции узкого канала с наклонной ОТЛ (со снятой верхней стенкой) с оцифрованными фрагментарными сетками: канальной (прямоугольной) (1), пристеночной, согласованной с поверхностью нижней стенки (2), и кромочной (3). Показано расположение системы декартовых координат $x, y, z$ с обозначением декартовых составляющих скорости $u, v, w$.

нат $x, y, z$ вводится с центром в середине нижней грани входного сечения облуненной секции канала (рис. 1).

Развитое турбулентное течение в облуненном канале описывается осредненными по Рейнольдсу уравнениями Навье-Стокса. Для замыкания системы уравнений движения применяется модель переноса сдвиговых напряжений (SST) 2003 г. в модификации, учитывающей влияние кривизны линий тока в рамках подхода Роди-Лешцинера-Исаева [11]. При решении конечно-объемных аналогов записанных в приращениях исходных уравнений применяются многоблочные вычислительные технологии, базирующиеся на разномасштабных пересекающихся сетках и гибридных сетках с неструктурированными вставками в местах наложения структурированных фрагментов. Используется метод коррекции градиента давления, основанный на сохранении расхода воздуха в канале. Применяется пакет VP2/3 (скорость-давление, 2D/3D) [12] и устанавливается сеточная сходимость расчетных результатов на сетках различной густоты и топологии. Использованные в численном исследовании многоблочные структурированные с наложением расчетные сетки состоят из трех оцифрованных на рис. 1 разномасштабных фрагментарных сеток. Общее количество расчетных ячеек многоблочной сетки составляет порядка 2.5 млн. Верификация модифицированной SST-модели и пакета VP2/3 на задачах турбулентного обтекания сферических и овально-траншейных лунок на стенке узкого канала проведена в [13].

Зависимости экстремальных характеристик течения и турбулентности, обезразмеренных по среднемассовой скорости, а также гидравлических потерь в периодической облуненной секции канала, определенных в рамках подхода [14] и отнесенных к потерям в гладком плоскопараллельном канале с индексом $p l$, от углов наклона ОТЛ на рис. 2 демонстрируют характерные диапазоны изменения, связанные с достижением экстремумов характеристик. Прежде всего отмечается диапазон малых углов наклона $\theta$ от 1 до 5-7, незначительно отличающихся от нуля. Все рассматриваемые на рис. 2 экстремальные величины (минимальные величины рассматриваются со знаком минус) декартовых составляющих скорости и энергии турбулентности довольно резко нарастают, достигая величин, близких к локальным экстремумам. Так, максимальные абсолютные величины скорости отрывного и вторичного течения $\left(-u_{\min }\right)$ и $\left(-w_{\min }\right)$ увеличиваются до 0.37 и 0.6 соответственно. Энергия турбулентности $k_{m}$ возрастает более чем в 1.5 раза по сравнению со случаем отрывного течения при $\theta=1^{\circ}$.

Следующий характерный диапазон углов $\theta$ простирается до $18^{\circ}$. В нем $\left(-w_{\min }\right)$, а также экстремальные скорости нисходящего и восходящего потоков $\left(-v_{\min }\right)$ и $v_{m}$ изменяются незначительно, сохраняя величины, близкие к $0.6,0.62$ и 0.22. Все остальные характеристики уменьшаются. Так, $\left(-u_{\min }\right)$ при $\theta=18^{\circ}$ достигает минимума порядка 0.2. Также вдвое до этой величины падает максимальная скорость вторичного течения $w_{m}$.

Основной диапазон углов наклона $\theta$, в котором наблюдается значительная интенсификация вторичного и возвратного течения, простирается от 18 до 65․ Величина $\left(-w_{\min }\right)$ в этом диапазоне возрастает почти двукратно, достигая максимума 1.16 при $\theta=50^{\circ},\left(-u_{\min }\right)$ увеличивается почти четырехкратно, достигая максимума, близкого к 0.8, при $\theta=52.5^{\circ}$. Наибольшие величины $w_{m}$ постепенно нарастают до 0.4 к $\theta=32.5^{\circ}$. Далее они сохраняются приблизительно на одном уровне до $\theta=40^{\circ}$, а затем немного увеличиваются и при больших углах наклона остаются неизменными на уровне 0.45 . Наибольшие скорости восходящих потоков $v_{m}$ достигают максимума, близкого к 0.55 , при $\theta=52.5^{\circ}$. Зависимости $-v_{\min }(\theta)$ и $k_{m}(\theta)$ разбиваются на два участка, на которых наблюдаются локальные максимумы и минимумы. Обе

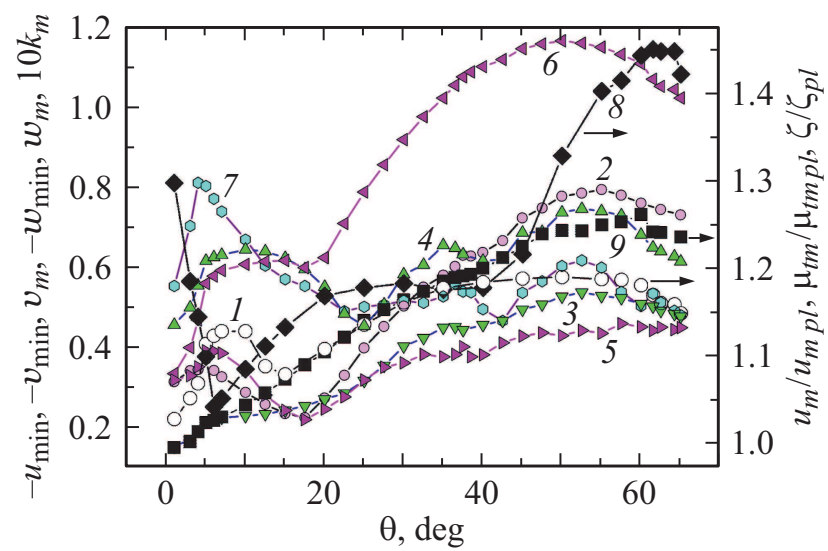

Рис. 2. Зависимости от угла наклона $\theta$ экстремальных и интегральных характеристик течения в облуненном канале: $u_{m} / u_{m p l}$ (1), (- $\left(-u_{\min }\right) \quad(2), v_{m} \quad(3), \quad\left(-v_{\min }\right) \quad(4), w_{m} \quad(5)$, $\left(-w_{\min }\right)(6), k_{m}(7), \mu_{t m} / \mu_{t m p l}(8)$ и $\zeta / \xi_{p l}(9)$. 

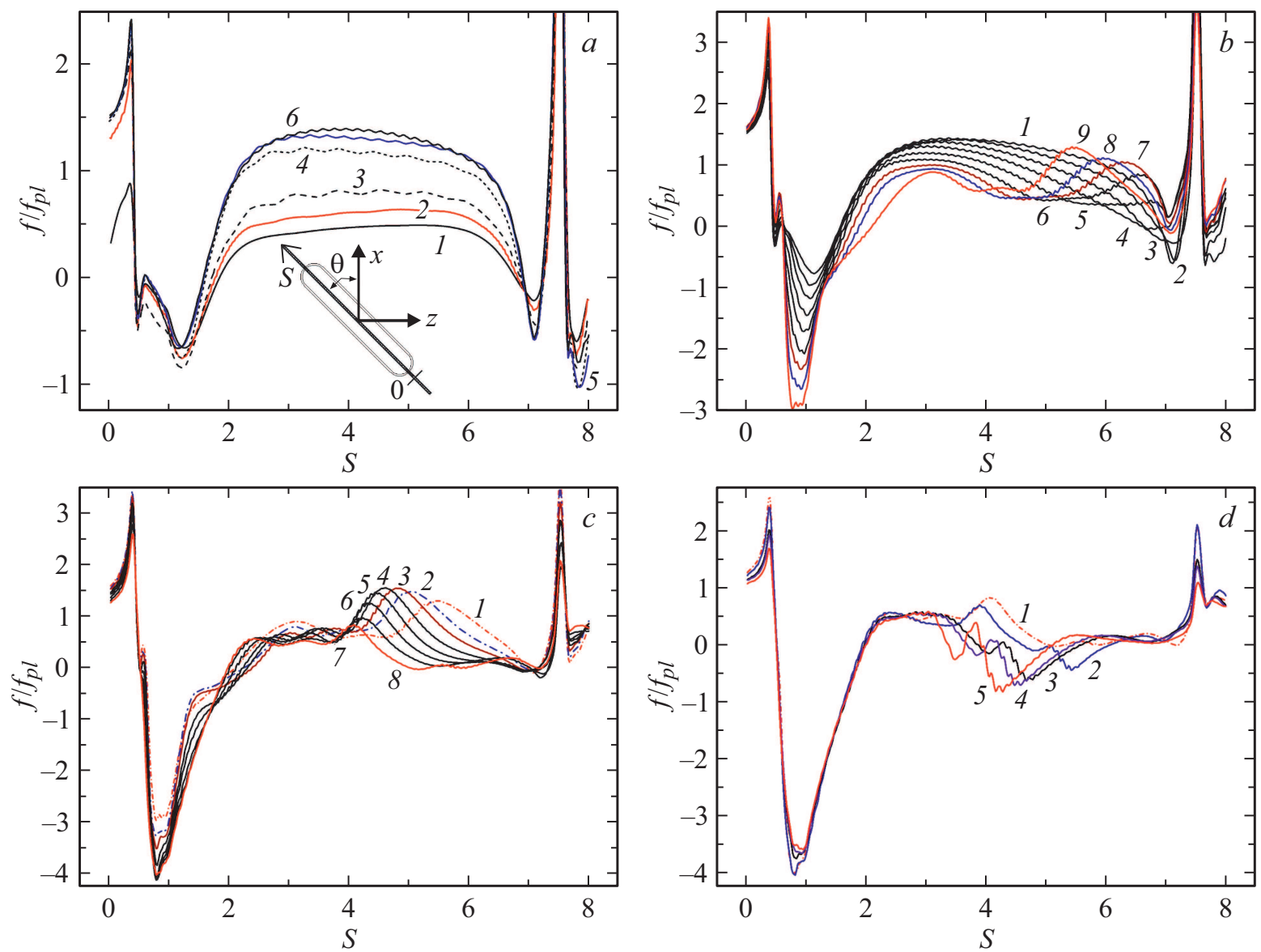

Рис. 3. Сравнение профилей относительного трения $f / f_{p l}$ в срединном сечении ОТЛ. $\left.\theta{ }^{\circ}{ }^{\circ}: a\right) 1-1,2-3,3-4,4-5,5-10$, $6-15 ; b) 1-17.5,2-20,3-22.5,4-25,5-27.5,6-30,7-32.5,8-35,9-37.5 ; c) 1-37.5,2-40,3-42.5$, $4-45,5-47.5,6-50,7-52.5,8-55 ; d) 1-55,2-57.5,3-61.5,4-62.5,5-65$.

характеристики достигают локальных минимумов при $\theta=22.5-25^{\circ}$ с некоторой задержкой по отношению к другим переменным. При этом минимальная величина $k_{m}(\theta)$ оказывается ниже уровня, зафиксированного при $\theta=1^{\circ}$, т.е. наблюдается эффект ламинаризации по мере интенсификации отрывного и закрученного потоков. Величина $\left(-v_{\min }\right)$ снижается до 0.45 , т.е. в 1.5 раза. С ростом $\theta$ при $\theta=35$ и $42.5^{\circ}$ обнаруживаются локальные максимумы и минимумы, причем $k_{m}$ значительно снижается (до 0.05) при $\theta=42.5^{\circ}$. При $\theta$ свыше $42.5^{\circ}$ обе переменные растут, достигая максимумов, равных 0.73 и 0.062 , при $\theta=52.5^{\circ}$.

Влияние $\theta$ кардинально влияет на интенсификацию отрывного и закрученного потоков в периодической ОТЛ. В двух диапазонах $\theta$ от 7 до $10^{\circ}$ и от 40 до $57.5^{\circ}$ наблюдается увеличение максимальной относительной скорости в ядре потока $u_{m} / u_{m p l}$ в 1.125 и в 1.2 раза. Здесь $u_{m p l}$ - величина максимальной скорости в гладкостенном узком канале. Максимум относительных гидравлических потерь $\xi / \zeta_{p l}$ наблюдается при $60^{\circ}$ и в 1.26 раза превышает потери в гладком канале $\left(\xi_{p l}\right)$. Максимум относительной вихревой вязкости $\mu_{t m} / \mu_{t m p l}$ при малых углах наклона линейно падает с 1.3 до 1.04, т.е. близок к 1. В дальнейшем с ростом $\theta$ до $20^{\circ}$ он нарастает линейно до 1.17 и остается на этом уровне до $40^{\circ}$. При средних и высоких $\theta$ свыше $40^{\circ} \mu_{t m} / \mu_{t m p l}$ быстро нарастает до величины 1.45 , соответствующей $60-63^{\circ}$, после чего начинает снижение. В диапазоне $\theta$ от 15 до $40^{\circ}$ кривые $u_{m} / u_{m p l}(\theta)$ и $\xi / \zeta_{p l}(\theta)$ совпадают.

Классификация режимов течения в лунке по мере ее наклона иллюстрируется профилями относительной $x$-составляющей трения $f / f_{p l}$ в срединном сечении лунки. Приведенные на рис. 3, $a$ зависимости $f / f_{p l}(S)$ показывают значительную интенсификацию течения в центральной части лунки по мере роста $\theta$ от 1 до $15^{\circ}$. Относительное трение уже при $\theta$ свыше $5^{\circ}$ превышает единицу, т.е. трение внутри лунки превосходит трение в гладком канале. На рис. $3, b$ рассмотрен следующий диапазон изменения $\theta$ от 17.5 до $37.5^{\circ}$, который характеризуется прежде всего интенсификацией течения во входной отрывной зоне. Относительное трение падает от -0.75 до -3. Длина отрывной зоны растет примерно от 1.07 до 1.6. В центральной зоне и на выходном участке лунки происходит перестройка течения. По мере роста $\theta$ монотонно уменьшается максимум относительного трения с 1.4 до 0.9, оставаясь на расстоянии примерно 
2.5 от входной кромки лунки. Третий диапазон изменения относительного трения представлен на рис. 3, и охватывает диапазон углов наклона от 40 до $55^{\circ}$. По мере роста наклона лунки усиливается интенсификация возвратного течения в передней части лунки и одновременно наблюдается изменение ее размера. Так, минимальное относительное трение снижается от -3.2 до -4.1. Длина отрывной зоны сначала достигает максимальной величины 1.85 при $\theta=42.5^{\circ}$, а дальше монотонно уменьшается до 1.96. Форма профиля $f / f_{p l}(S)$ становится все более наполненной. Наиболее сложная перестройка течения происходит в центральной части лунки в зоне в целом безотрывного течения. Четвертый диапазон изменения углов наклона лунки представлен на рис. $3, d$ и охватывает диапазон углов наклона от 55 до $65^{\circ}$. Интенсификация отрывного течения во входной части лунки начинает ослабевать. Так, минимальное относительное трение возрастает от -4 до -3.6 . Длина отрывной зоны немного сокращается (от 1.46 до 1.36). Наиболее интересные структурные метаморфозы происходят в центральной части лунки, где при больших углах наклона формируется зона интенсивного возвратного течения.

Таким образом, наклон овально-траншейной лунки в коридорном пакете на стабилизированном гидродинамическом участке узкого канала оказывает кардинальное воздействие на аномальную интенсификацию турбулентного отрывного и закрученного потоков в лунке, приводя к трех-четырехкратному снижению относительного отрицательного трения в диапазоне изменения углов от 40 до $60^{\circ}$, увеличению наибольшей скорости возвратного и вторичного течения до 0.8 и 1.18 по отношению к среднемассовой скорости потока в канале, ускорению канального потока в 1.2 раза по сравнению с максимальной скоростью в плоскопараллельном канале.

\section{Финансирование работы}

Работа выполнена при поддержке Российского научного фонда (грант № 19-19-00259).

\section{Конфликт интересов}

Авторы заявляют, что у них нет конфликта интересов.

\section{Список литературы}

[1] Вихревые технологии для энергетики / Под общ. ред. акад. А.И. Леонтьева. М.: Изд. дом МЭИ, 2017. 500 с.

[2] Rashidi S., Hormozi F., Sunden B., Mahian O. // Appl. Energy. 2019. V. 250. P. 1491-1547.

[3] Rutten M., Krenkel L. // Notes Num. Fluid Mech. Multidiscipl. Des. 2010. V. 112. P. 555-562.

[4] Henry F.S., Pearcey H.H. // AIAA J. 1994. V. 32. P. 2415-2425.

[5] Исаев С.А., Леонтьев А.И., Баранов П.А. // Письма в ЖТФ. 2000. Т. 26. В. 1. C. 30-37.
[6] Исаев С.А., Леонтьев А.И., Митяков А.В., Пышный И.А. // Инж.-физ. журн. 2003. Т. 76. № 2. С. 31-34.

[7] Гувернюк С.В., Чулюнин А.Ю. // Письма в ЖТФ. 2019. T. 45. B. 17. C. $43-46$.

[8] Исаев С.А., Леонтьев А.И., Гульцова М.Е., Попов Ю.А. // Письма в ЖТФ. 2015. Т. 41. В. 12. С. 89-96.

[9] Isaev S., Gritckevich M., Leontiev A., Popov I. // Acta Astronaut. 2019. V. 163. Pt A. P. 202-207.

[10] Isaev S.A., Gritckevich M.S., Leontiev A.I., Milman O.O., Nikushchenko D.V. // Int. J. Heat Mass Transfer. 2019. V. 145. P. 118737 (1-13).

[11] Isaev S.A. // AIP Conf. Proc. 2018. V. 2027. P. 020015 (1-7).

[12] Исаев С.А., Баранов П.А., Усачов А.Е. Многоблочные вычислительные технологии в пакете VP2/3 по аэротермодинамике. Саарбрюккен: LAP LAMBERT Academic Publ., 2013. $316 \mathrm{c}$.

[13] Isaev S., Leontiev A., Chudnovsky Y., Nikushchenko D., Popov I., Sudakov A. // Energies. 2019. V. 12. P. 1-24.

[14] Isaev S.A., Kornev N.V., Leontiev A.I., Hassel E. // Int. J. Heat Mass Transfer. 2010. V. 53. P. 178-197. 